\title{
Repräsentative Einbände - die Sammlung Boehringer
}

Im April 2019 gelangte eine außergewöhnliche Sammlung bibliophiler Bücher in den Besitz der Württembergischen Landesbibliothek Stuttgart. Aus dem Nachlass von Hans Boehringer (gest. 2003) stammen 77 Bände, die insbesondere den Bestand buchgeschichtlich relevanter Einbände ergänzen.

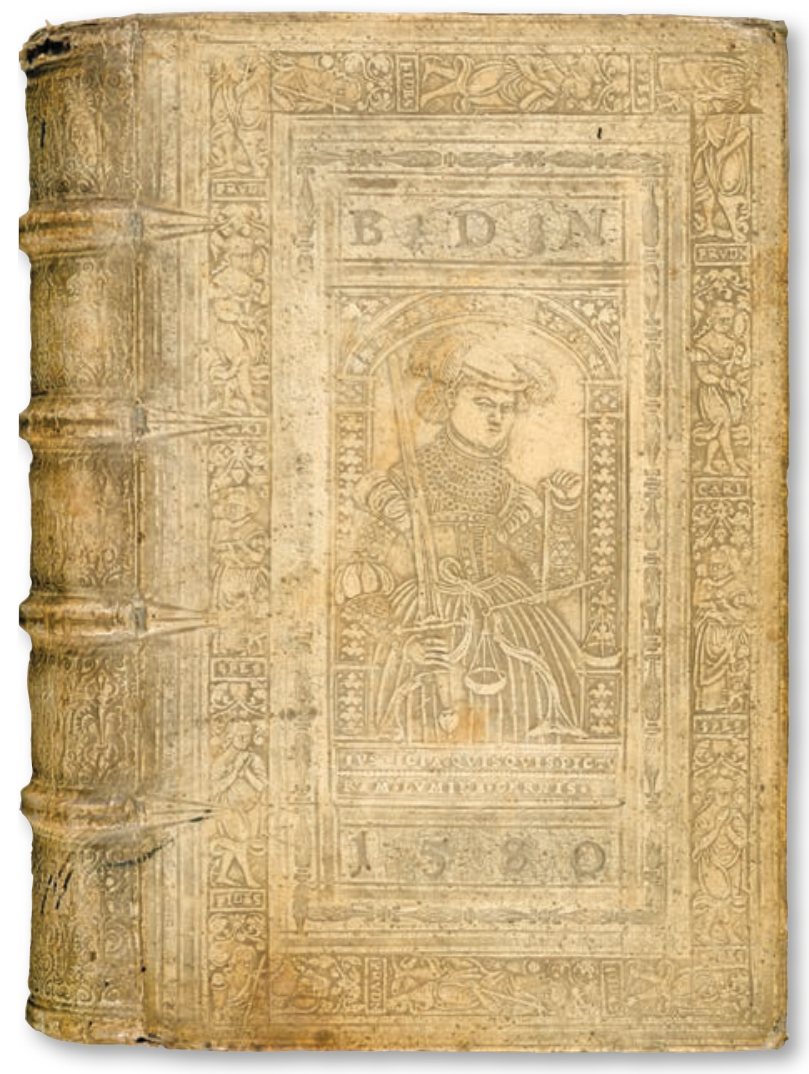

Abb. 1: Blindgeprägter Schweinslederband

Fast die Hälfte der Werke wurde im 18. Jahrhundert gedruckt; 24 Bände sind dem 17. Jahrhundert zuzuordnen und der Rest verteilt sich auf das 16., 19. und 20. Jahrhundert. Drei Handschriften entstanden im 18. Jahrhundert. Die Frankophilie des Vorbesitzers wirkte sich in der sprachlichen Verteilung aus. Allein 51 Bände sind in französischer Sprache gehalten, vier weitere zweisprachig in Latein und Französisch. 13 sind in Latein verfasst. Die restlichen Ausgaben verteilen sich auf Italienisch, Deutsch und Englisch. Die meisten Bücher wurden wegen ihrer Ausstattung mit reich verzierten Einbänden sowie Illustrationen, einige auch wegen damit verbundener besonderer Provenienzen erworben. 55 Einbände wurden in Frankreich hergestellt. Diese verteilen sich auf Wappen-Supralibros-Einbände (46), marmorierte Ledereinbände (8) sowie je einen Einband im Semis-Stil bzw. im historistischen oder im Neobarock-Stil. Man findet aber auch sechs blindgeprägte Schweinsledereinbände des 16. Jahrhunderts, einen goldgeprägten Kalbsledereinband des späten 16. oder frühen 17. Jahrhunderts sowie englische, italienische, österreichische und deutsche Wappen-SupralibrosEinbände in der Sammlung. Hinzu kommen zwei Miniaturbücher und ein bibliophiler Verlagseinband.

Die Interessen der meist aus dem Hochadel bzw. aus höfischen Kreisen stammenden Vorbesitzer spiegeln sich im inhaltlichen Profil der Sammlung wider. Von Überschneidungen zum Sammelinteresse Boehringers ist auszugehen. 38 Bände kann man dem lyrisch-philologischen Bereich zurechnen. Dazu zählen alle mehrbändigen Werke und originalsprachliche Editionen lateinischer Klassiker oder deren französische Übersetzung. Acht Bände betreffen Ereignisse aus dem Leben von Herrschern oder für das höfische Leben besonders relevante Themen wie Malerei, Reiten oder Geschmack. Allein sechs liturgische Bücher für die Partizipation am Gottesdienst in der Pariser Hofkapelle liegen vor. Der Rest verteilt sich auf Werke zur Geschichte, Theologie, Politik, Philosophie, Wirtschaft, Geographie, Botanik oder alltägliche Hilfsmittel wie ein Notizbuch oder einen Almanach.

Einige Beispiele mögen Struktur und Bedeutung der Sammlung veranschaulichen.

Zu den älteren Stücken zählt eine 1580 in Genf gedruckte Ausgabe der lateinischen Reden Ciceros. Dieses Exemplar weist einen gut erhaltenen Schweinslederband mit blindgeprägten Verzierungen einer anonymen deutschen Werkstatt auf (EBDB w007428). Gut erkennbar sind auf dem noch im Druckjahr hergestellten Einband PlattenMotive mit Justitia bzw. Fortuna. 


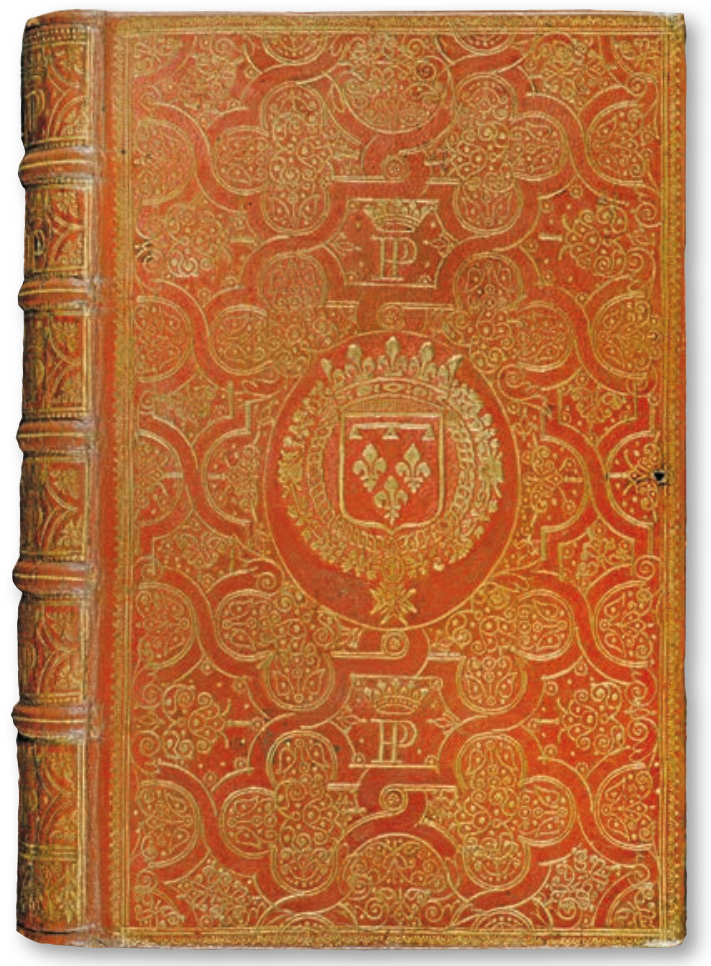

Abb. 2: Wappen-Einband Herzog Philipps II.
Teilweise in lyrischer Form enthält eine Handschrift unter dem Titel „Philippiques en cinq odes" Nachrichten aus dem Leben des Bourbonen Philipp II., des Herzogs von Orléans (1674-1723). Er übte für den unmündigen späteren König Ludwig XV. von 1715 bis 1723 die Regentschaft aus. Der mit in sich verschlungenem Bandwerk und filigraner floraler Ornamentik verzierte Einband weist Philipps Monogramm PP sowie seine persönliche Variante des Lilien-Wappens der Bourbonen als Supralibros auf.

Stilistisch verwandt ist der Einband für eine 1728 gedruckte Ausgabe der Liturgie während der Karwoche. Das lateinisch-französische Werk wurde Königin Maria von Frankreich (1703-1768) gewidmet. Die Tochter des späteren polnischen Königs Stanisław I. Leszcyński hatte 1725 den französischen König Ludwig XV. geheiratet und prägte ihre lange Amtszeit auch durch ihre tiefe Frömmigkeit. Das Supralibros deutet die Intention damaliger
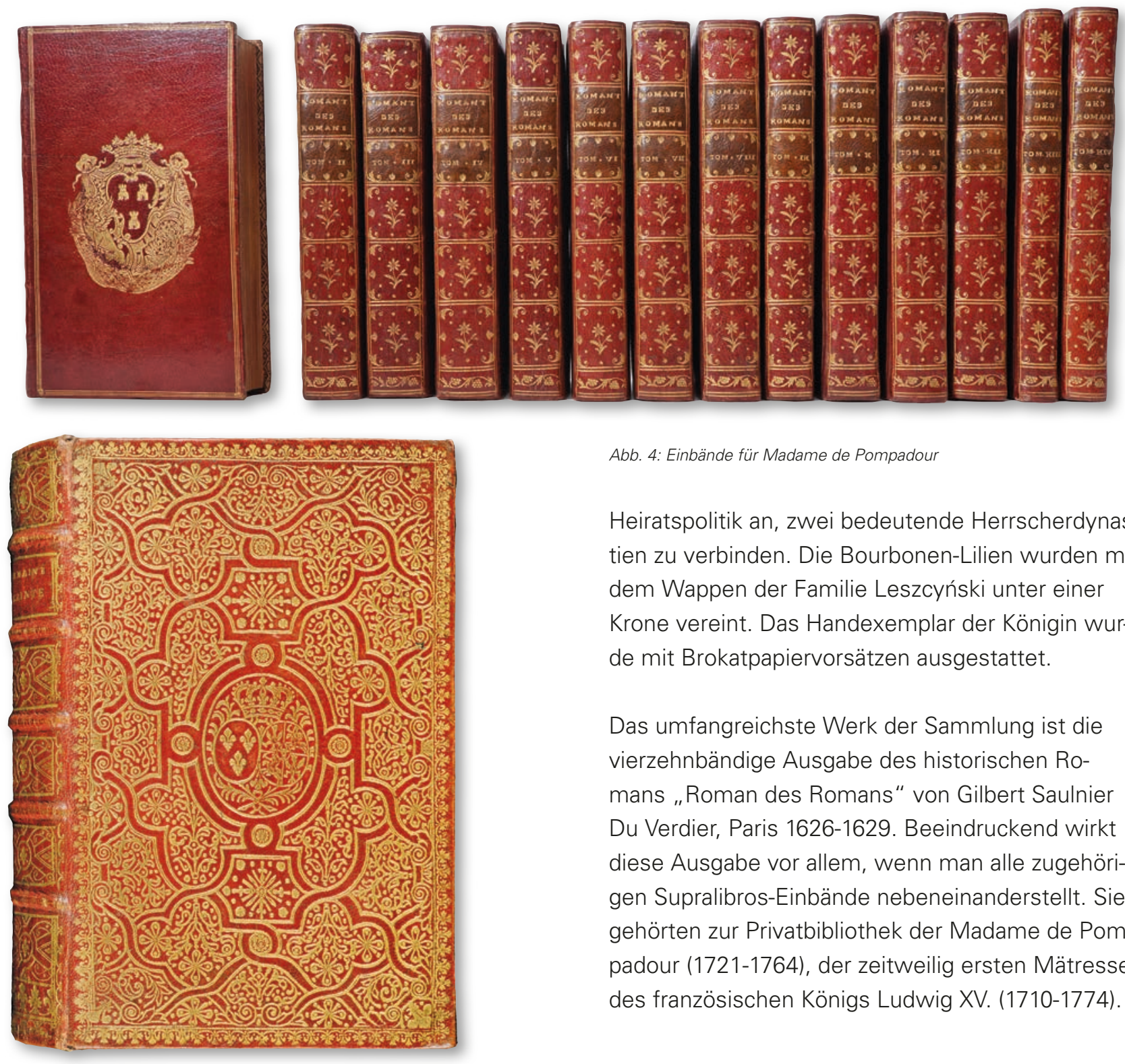

Abb. 4: Einbände für Madame de Pompadour

Heiratspolitik an, zwei bedeutende Herrscherdynastien zu verbinden. Die Bourbonen-Lilien wurden mit dem Wappen der Familie Leszcyński unter einer Krone vereint. Das Handexemplar der Königin wurde mit Brokatpapiervorsätzen ausgestattet.

Das umfangreichste Werk der Sammlung ist die vierzehnbändige Ausgabe des historischen Romans "Roman des Romans" von Gilbert Saulnier Du Verdier, Paris 1626-1629. Beeindruckend wirkt diese Ausgabe vor allem, wenn man alle zugehörigen Supralibros-Einbände nebeneinanderstellt. Sie gehörten zur Privatbibliothek der Madame de Pompadour (1721-1764), der zeitweilig ersten Mätresse des französischen Königs Ludwig XV. (1710-1774). 


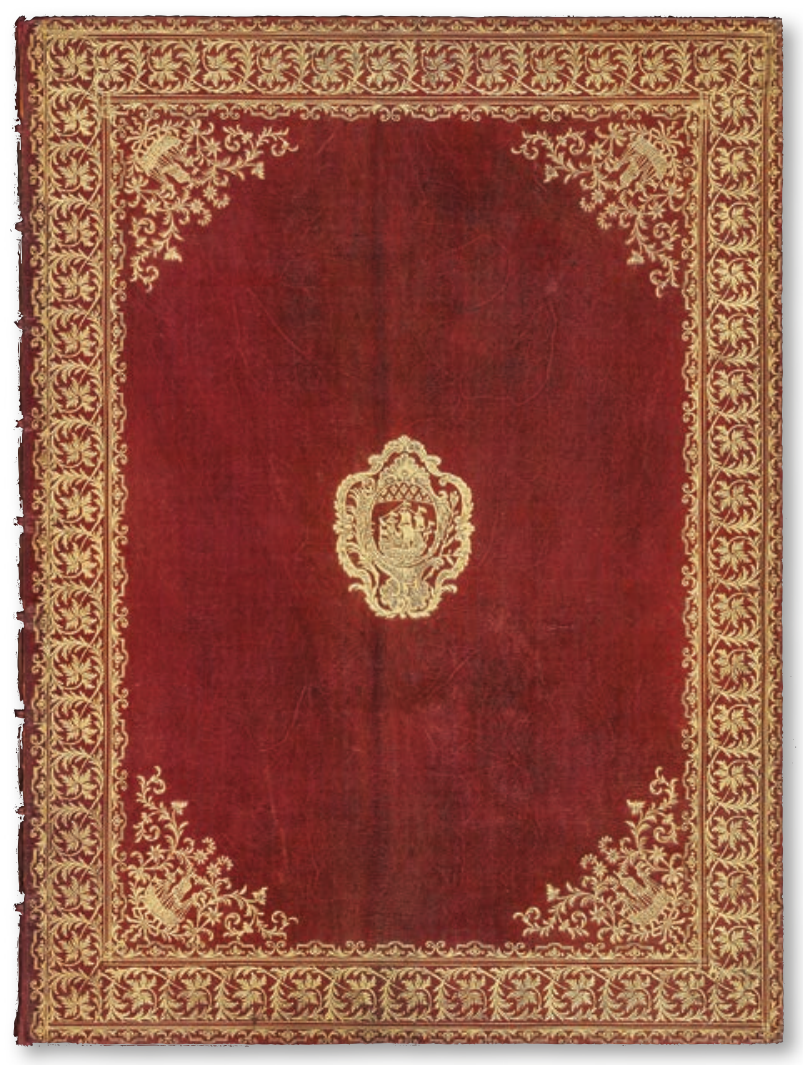

Abb. 5: Bildband zur Prinzen-Hochzeit

Die Kupferstichtafeln eines großformatigen Bildbands präsentieren detailreich alle Festwagen, die 1747 bei der Hochzeitsfeier des französischen Thronanwärters Louis Ferdinand de Bourbon (1729-

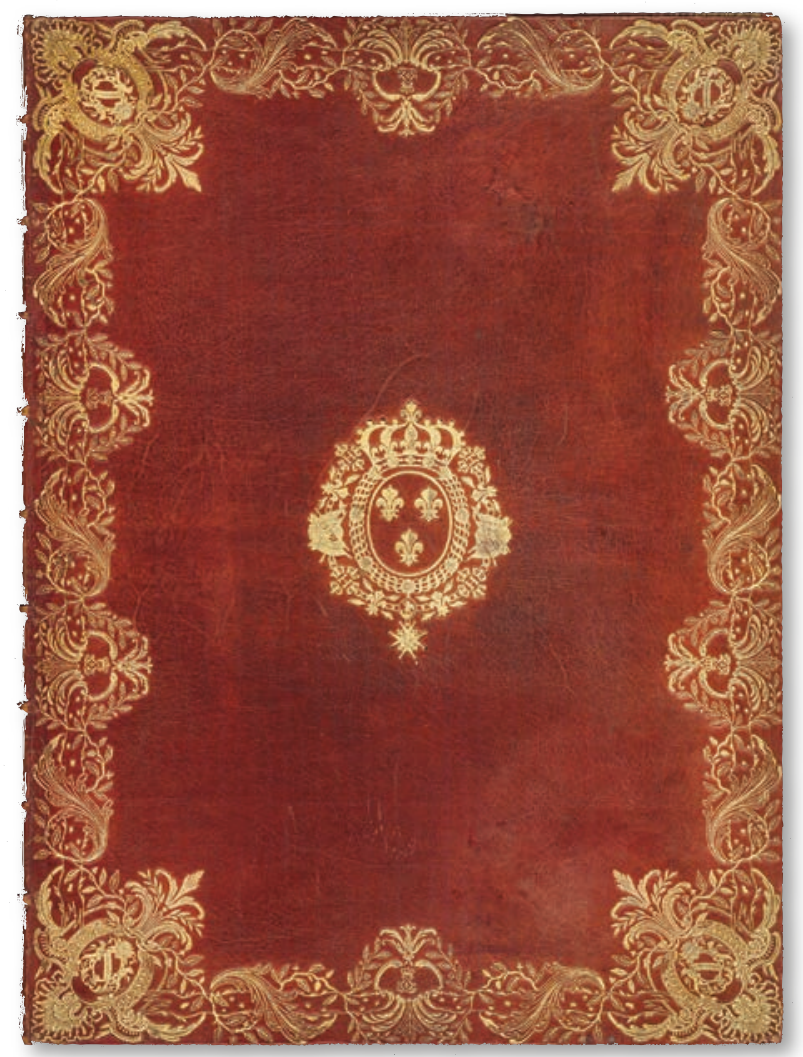

Abb. 6: Festbuch für Ludwig XV.
1765) mit der sächsisch-polnischen Prinzessin Maria Josepha (1731-1767) fuhren. Der festliche Maroquin-Einband des königlichen Buchbinders Antoine-Michel Padeloup (1685-1758) verwendet das Motiv eines Schiffes mit den Bourbonen-Lilien. Dies erschien als Symbol für den Eintritt in den Ehestand geeignet und stellte den Bezug zum traditionellen Delphin-Motiv des Dauphin her.

Ebenfalls von Padeloup stammt der Einband für einen Tafelband mit Darstellungen der Feierlichkeiten während des Aufenthaltes des französischen Königs Ludwig XV. in der Stadt Straßburg im Oktober 1744. Die Kupferstiche wurden vom Straßburger Künstler Johann Martin Weis (1711-1751) erstellt. Der Einband zeigt das Wappen des Königs und in den Eckfeldern das Stadtwappen Straßburgs.

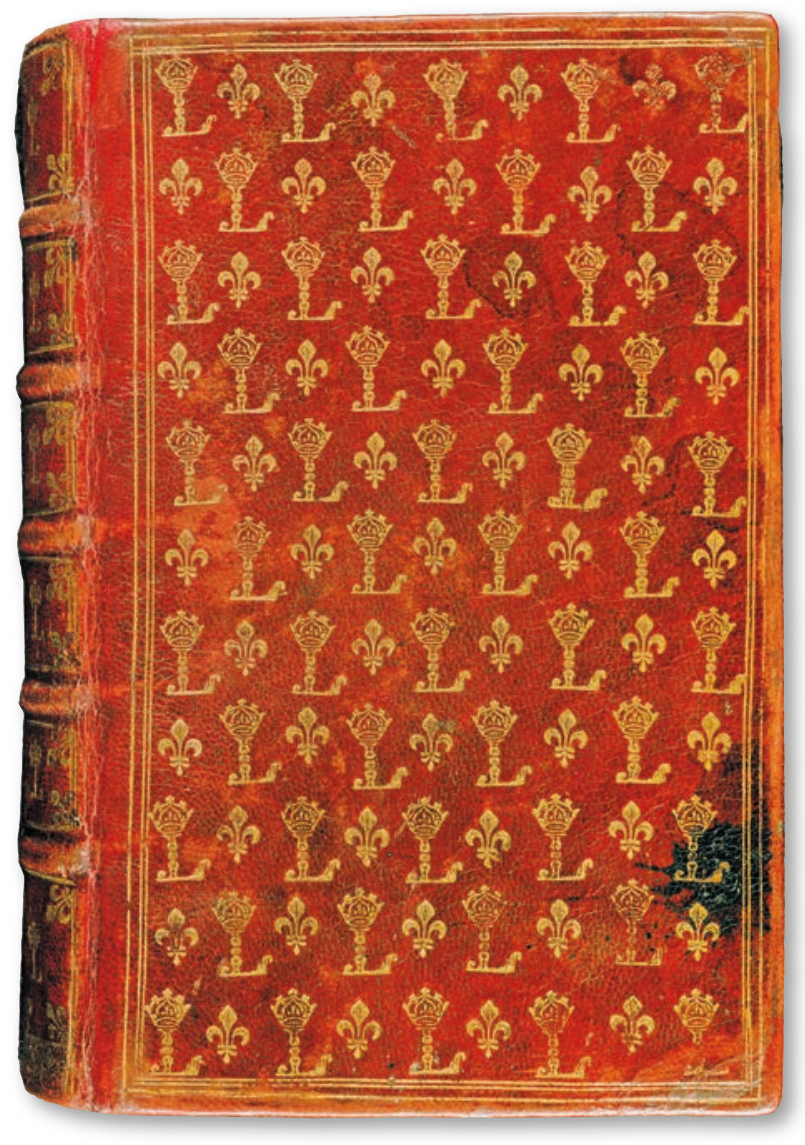

Abb. 7: Buch des Sonnenkönigs

Aus dem Besitz des Sonnenkönigs Ludwig XIV. (1638-1715), des Urgroßvaters Ludwigs XV., stammt ein Stundenbuch für die Karwoche, das ca. 1695 in Paris gedruckt wurde. Der Einband wurde im barocken Semis-Stil dekoriert: Wie bei einem Blumenbeet wurden die Deckel mit zwei jeweils im Wechsel verwendeten Stempeln versehen. Die Bourbonen-Lilie galt dabei als dynastisches 
Symbol, das große L mit Krone als Monogramm des Königs.

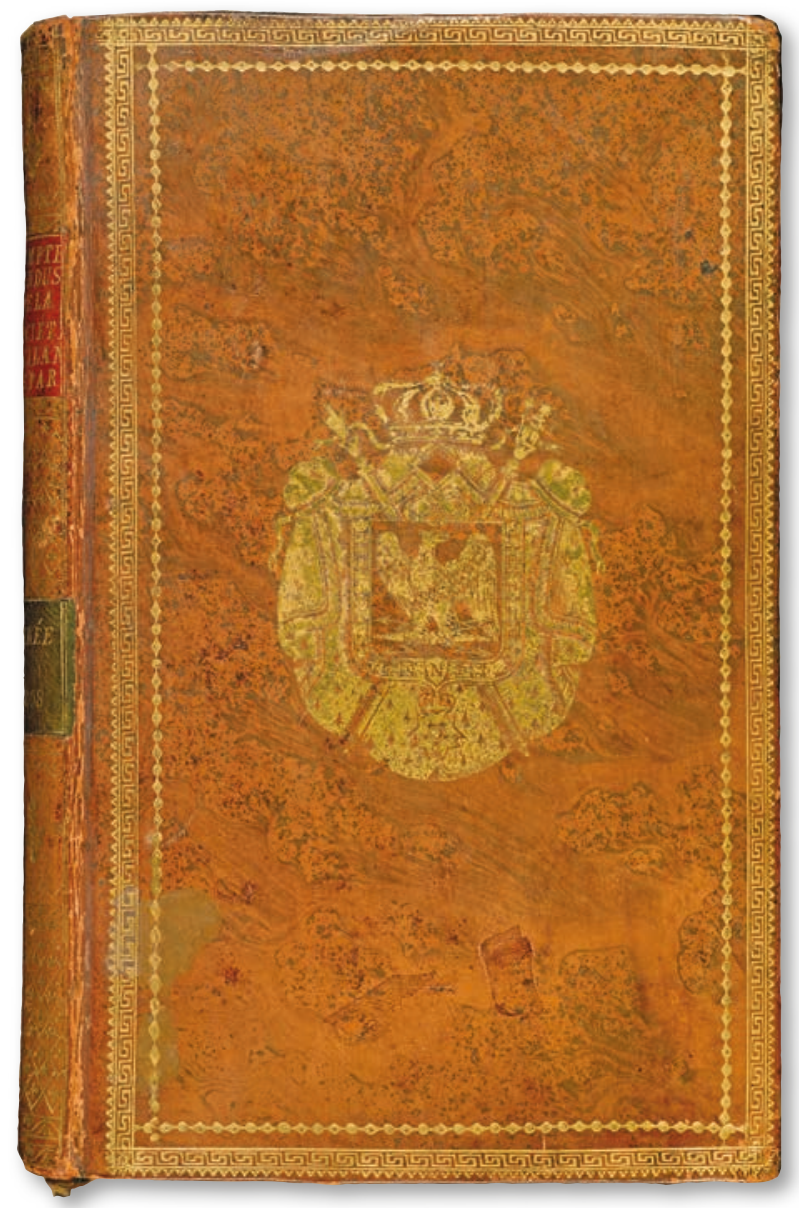

Abb. 8: Wappen Napoleons

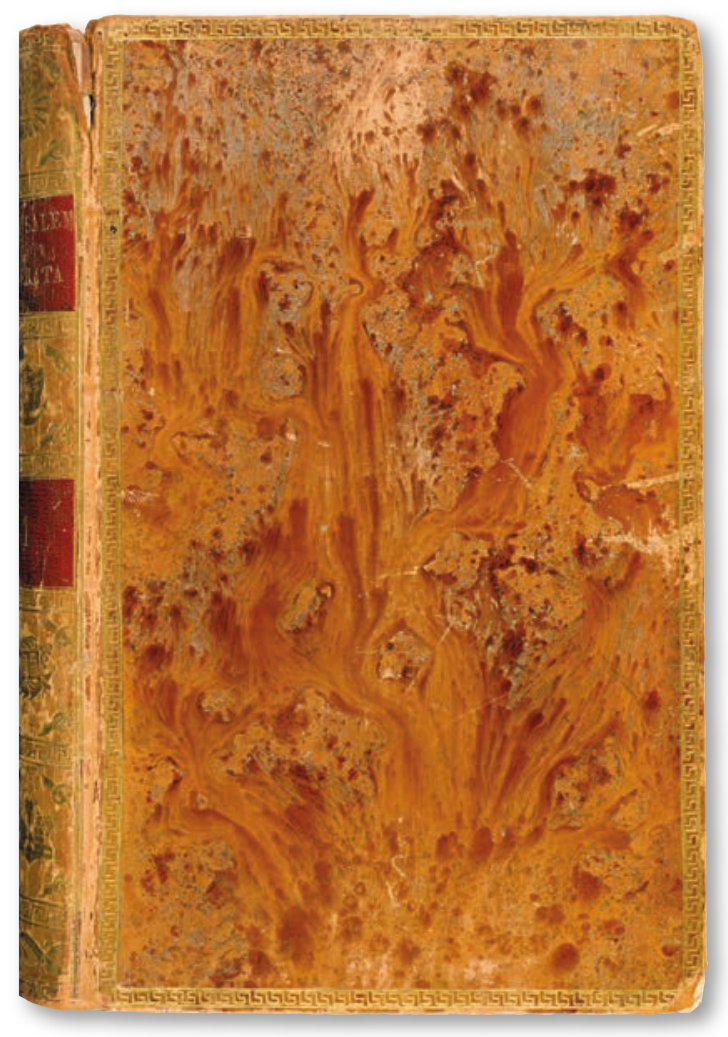

Nicht zum Hochadel gehörig, aber von den historischen Nachwirkungen her wohl der bedeutendste Vorbesitzer war Kaiser Napoleon I. (1769-1821). Dass es sich bei dem marmorierten Kalbslederband mit kaiserlichem Supralibros ausgerechnet um einen Tätigkeitsbericht der Philanthropischen Gesellschaft von Paris aus dem Jahr 1808 handelt, entbehrt angesichts seiner Expansionspolitik nicht einer gewissen Ironie. Durch Interessen, Motivation und Symbolik wollte sich Napoleon allerdings von den Bourbonen deutlich unterscheiden.

1792, also mitten in den Revolutionswirren, erschien in Paris eine originalsprachliche Edition des italienischen Kreuzzugs-Epos' "La Gerusalemme liberata" von Torquato Tasso (1544-1595). Das Werk wurde in zwei Bänden mit marmoriertem Kalbsledereinband in Holz-Anmutung gebunden.

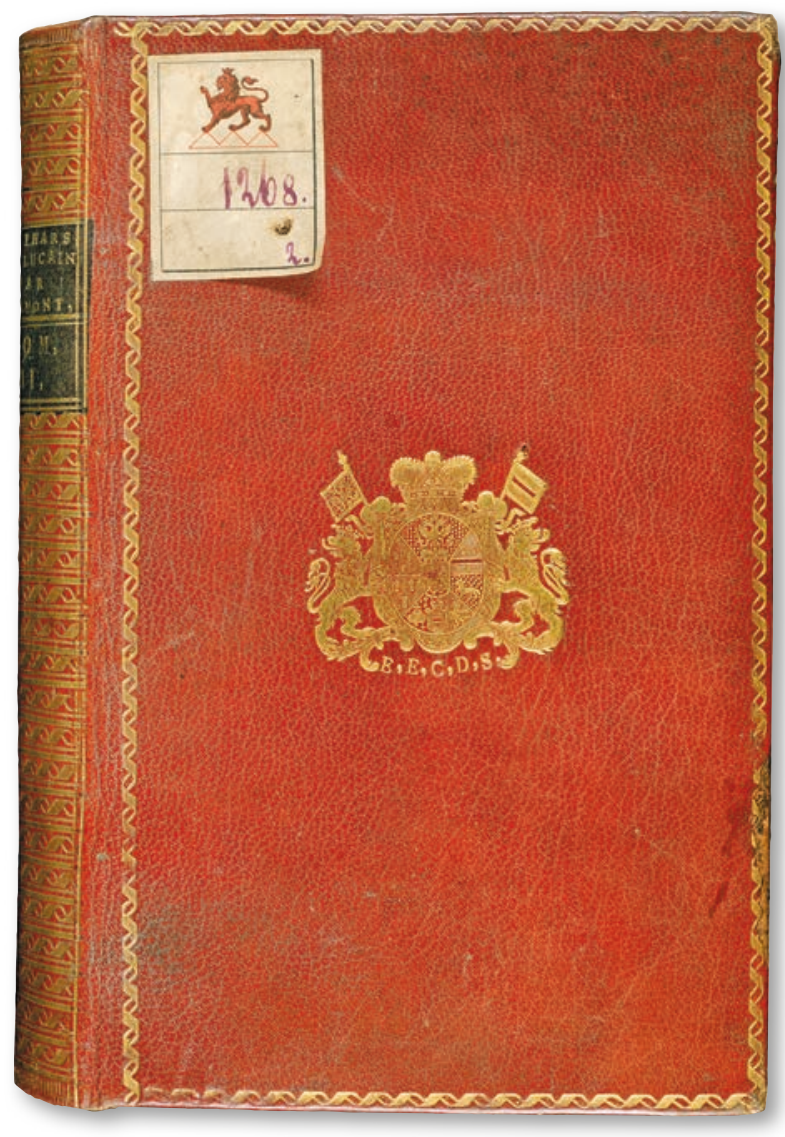

Abb. 10: Schönborn-Supralibros

Eine 1766 in Paris gedruckte französische Edition von Lukans lateinischem Epos "De bello civili" wurde durch ein Wappen-Supralibros mit Initialen (E. E. C. D. S.) verziert. Die roten Maroquin-Bände gehörten zur Privatbibliothek des Grafen Eugen Franz Erwein von Schönborn-Buchheim (1727- 
1801). Der Neffe des Würzburger Fürstbischofs war kaiserlicher Geheimrat und Kämmerer. Die Bücher aus seiner Schlossbibliothek in Göllersdorf (Niederösterreich) gelangten wohl nach 1945 teilweise in Streubesitz.

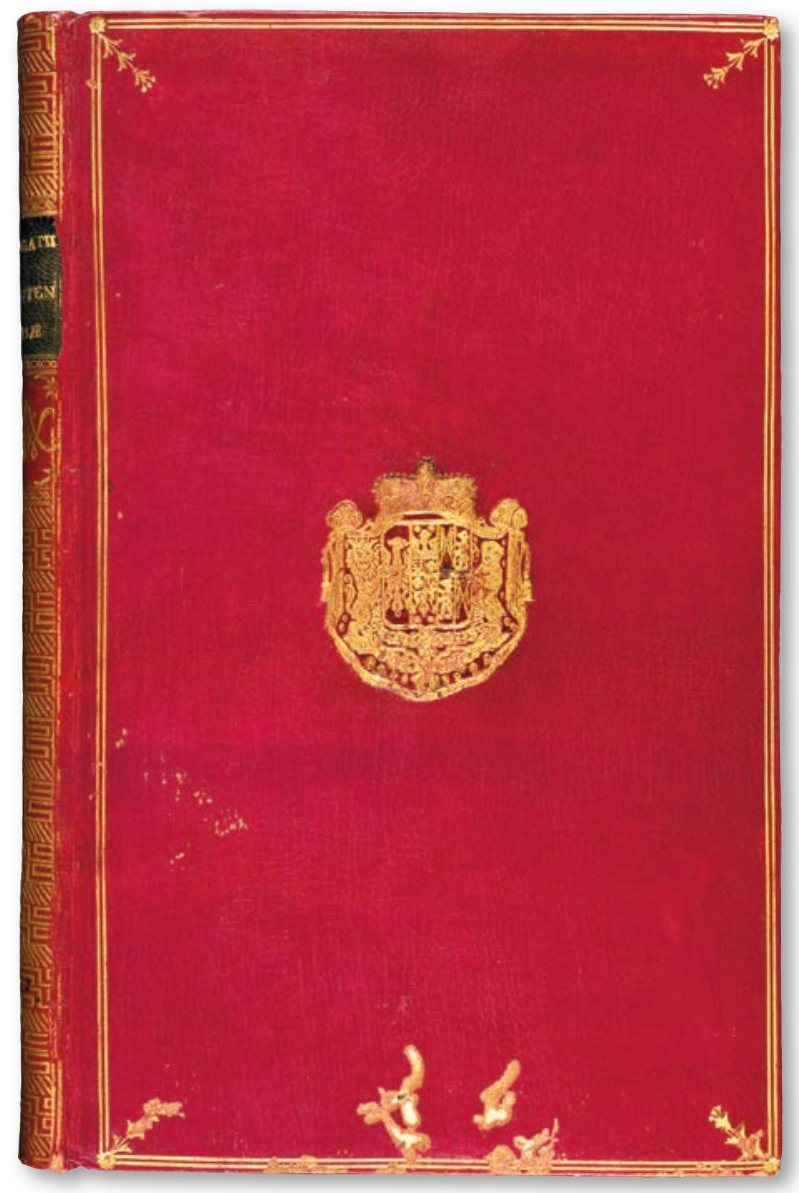

Abb. 12: Bayerischer Wappen-Supralibros-Einband

Etwas weniger bedeutend ist die mit nur drei Teilbänden vertretene Werkausgabe des französischen Dramatikers Néricault Destouches (1680-1754). Allerdings deutet das Wappen-Supralibros des bayAbb. 11: Einband für den Fürsten Kaunitz-Rietberg erischen Kurfürsten Maximilian III. Joseph (17271777) darauf hin, in welchem Maße französische Literatur von deutschen Barockfürsten rezipiert wurde.

sche Handschrift mit ausgewählten Zitaten aus den Sentenzen des Horaz, die auf 1786 datiert ist. Sie wurde für den persönlichen Gebrauch des Fürsten Wenzel Anton von Kaunitz-Rietberg (1711-1794) erstellt. Kaunitz war als Diplomat und Staatsmann für zahlreiche Verwaltungsreformen und außenpolitische Entscheidungen Österreichs unter Maria Theresia sowie Joseph II. verantwortlich. Der Maroquin-Einband mit Seiden-Vorsatz weist ein Christian Herrmann Supralibros mit dem fürstlichen Wappen auf.

Die kleine, aber zahlreiche sehr bedeutende Stücke umfassende Sammlung Boehringer veranschaulicht exemplarisch Bücher als Repräsentationsobjekte im europäischen Adel des 17. und 18. Jahrhunderts. 